\title{
Algunas notas sobre el léxico veracruzano en la época colonial (1650-1700). La presencia de americanismos en textos novohispanos del Golfo de México
}

\section{Some Notes on Veracruz Lexicon during Colonial Period (1650-1700). The Presence of Americanisms in New Spain Texts from Gulf of Mexico}

\author{
María Adriana Gutiérrez Bello [adrianagtzbello@gmail.com] \\ Universidad Autónoma de Querétaro, México
}

\section{RESUMEN}

Este trabajo pretende aportar datos para ahondar en el conocimiento del léxico novohispano mediante el análisis de algunas unidades susceptibles de ser consideradas americanismos léxicos que aparecen en 18 textos producidos durante la segunda mitad del siglo XVII en el estado de Veracruz incluidos en los Documentos lingüísticos de la Nueva España. Golfo de México (Melis y Rivero Franyutti 2008). Se busca aprovechar así este relevante corpus documental, editado con criterios filológicos que facilitan el proceso de investigación en distintas disciplinas, para extraer información sobre el español en uso en una región y una sincronía escasamente abordadas hasta el momento por los especialistas.

\section{Palabras clave}

Historia del español en América; siglo XVII; léxico novohispano; americanismo léxico; indigenismo

\begin{abstract}
This paper aims to provide data to deepen the knowledge of the Novohispanic lexicon through the analysis of some units that can be considered lexical Americanisms, wich appear in 18 texts produced during the second half of the 17th century in the State of Veracruz (Documentos lingüísticos de la Nueva España. Golfo de México, Melis and Rivero Franyutti, 2008). The goal is to take advantage of this relevant corpus, edited with philological criteria that facilitate the research process in different disciplines, to extract data on Spanish in use in a region and a synchronicity scarcely studied by the specialists.
\end{abstract}

\section{KEYWORDS}

History of Spanish in America; 17th century; Novohispanic lexicon; lexical Americanism; indigenism

RECIBIDO 2018-03-20; ACEPTADO 2018-05-18 


\section{Introducción}

El siglo XVII forma parte de un periodo de esplendor para el español en la Península Ibérica durante el cual se consolida su predominio en diversos ámbitos -político, geográfico, social y cultural-. Sin embargo, en América, el panorama es distinto: constituye el medio de comunicación de la sociedad criolla, de la administración pública, de la ciudad, en tanto que en las zonas rurales todavía es un idioma minoritario en un contexto de gran diversidad lingüística (Ramírez Luengo 2007: 26-27). Tomando en cuenta estos antecedentes, llama la atención la escasez de obras basadas en documentos de la época sobre las características de la lengua, y en particular del léxico, a lo largo de este complejo proceso de arraigo.

Apenas a fines del siglo pasado, el léxico histórico del periodo virreinal comienza a ser un tema de interés para los especialistas, antes concentrados en los primeros contactos del español con la realidad americana. Algunos ejemplos de esta tendencia son los trabajos de Franco Figueroa (1991) acerca del vocabulario hispanoamericano durante los siglos XVI y XVII y de Frago (1999) en torno a la influencia del origen de los migrantes en la configuración del español en América, así como el exhaustivo proyecto de Company y Melis (2002) relativo al léxico histórico de México y las observaciones de Quirós García y Ramírez Luengo (2015) respecto al español de Yucatán de 1650 a 1800. A pesar de las aportaciones de estos autores, es mucho lo que queda por documentar en relación con este episodio de la historia del español en América.

Por otro lado, el estado de Veracruz es una zona dialectal que a su vez se divide en tres regiones: sur, de Minatitlán a Tlacotalpan; centro $^{1}$, del puerto de Veracruz a Misantla, y norte, de Papantla a Tempoal. Si bien desde estudios dialectológicos previos al Atlas Lingüístico de México, se distinguió a la entidad de las zonas yucateca-campechana y tabasqueña con base en los rasgos fonéticos, morfosintácticos y lexicológicos más representativos de cada variedad (Lope Blanch 1970: 9-11), hasta el momento, no ha habido trabajos que ahonden en los resultados vinculados con el habla veracruzana.

Es notable la ausencia de investigaciones enfocadas en la historia del español de Veracruz, en especial durante la Colonia, considerando la relevancia de la región en dicho periodo, pues en ella se fundaron algunas de las poblaciones más antiguas de españoles, además de albergar el mayor puerto de la Nueva España - principal vía de ingreso de viajeros, inmigrantes y esclavos al virreinato- y grandes asentamientos afromestizos² (Aguirre Beltrán 1989: 154-156). De ahí que resulte de gran interés documentar y analizar las huellas de este crisol cultural en el léxico del español en uso en la entidad.

Los Documentos lingüísticos de la Nueva España. Golfo de México (Melis y Rivero Franyutti 2008) -en adelante, DLNEGM- constituyen una fuente idónea para llevar a cabo estas tareas, pues se trata de un corpus integrado por materiales de archivo, elegidos por su cercanía a la

1 La región centro, por cierto, es considerada como "la más homogénea y, a la vez, el foco lingüístico irradiador con respecto a las otras dos" (Moreno de Alba 1976: 352).

2 El jarocho, "mezcla de india y negro" -o viceversa-, es el habitante prototípico de la antigua región del Sotavento que hoy corresponde tanto a Veracruz como a parte de los estados de Tabasco y Oaxaca; la zona también se asocia con los rebeldes cimarrones encabezados por Yanga, quien a comienzos del siglo XVII funda la primera población en la que es abolida la esclavitud en América: San Lorenzo de los Negros o San Lorenzo Cerralvo (INAFED 2010: en línea). 
oralidad y editados con criterios filológicos fiables, accesible para investigadores de diversas disciplinas ${ }^{3}$.

\section{Propósitos del estudio y metodología}

Con el estudio, se pretende profundizar en el conocimiento del léxico en uso en Veracruz durante la época colonial, concretamente durante la segunda mitad del siglo XVII, además de buscar posibles rasgos de especifidad dialectal en el nivel de la lengua señalado (americanismos léxicos) y ejemplificar las clases de elementos que van a configurar dicha especifidad (tipos de americanismos léxicos).

Para ello, se ha analizado el vocabulario presente en los 18 textos producidos entre 1650 y 1700 en la entidad que se incluyen en los DLNEGM, los cuales pertenecen a los fondos Inquisición y Hospitales del Archivo General de la Nación, principal acervo histórico de México. Entre estos documentos, predominan los procedentes del puerto de Veracruz y las denuncias, como se observa a continuación. (TABLAS 1 y 2 )

\begin{tabular}{|l|c|}
\hline ProcedenCIA & NúM. De DOCUMENTOS \\
\hline Veracruz & 8 \\
\hline Orizaba & 4 \\
\hline Xalapa & 3 \\
\hline Córdoba & 2 \\
\hline Santa Ana & 1 \\
\hline Total & $\mathbf{1 8}$ \\
\hline
\end{tabular}

Tabla 1. Procedencia de los DLNEGM producidos en Veracruz entre 1650 y 1700.

\begin{tabular}{|c|c|}
\hline TIPO TEXTUAL & NúM. DE DOCUMENTOS \\
\hline Denuncia & 7 \\
\hline Testimonio & 6 \\
\hline Informe & 2 \\
\hline Carta & 1 \\
\hline Petición & 1 \\
\hline Inventario & 1 \\
\hline TOTAL & 18 \\
\hline
\end{tabular}

Tabla 2. Tipo textual de los DLNEGM producidos en Veracruz entre 1650 y 1700.

3 Es el segundo corpus creado con estas características en México. El primero es el coordinado por Company (1994) que cubre el mismo periodo, pero en la región del Altiplano Central. 
El estudio se enfoca en los elementos identificados en los documentos como americanismos -es decir, las voces diatópicamente marcadas que caracterizan a las variedades del español en América-. Al ser una noción fundamental para desarrollar el análisis propuesto, se ha decidido tomar como punto de partida una definición clara del americanismo que sirva como marco conceptual en "la búsqueda de estos elementos diferenciales" (Quirós García y Ramírez Luengo 2015: 187). Es importante destacar que hasta el momento no hay consenso con respecto a este tema, sino aportes teóricos y metodológicos muy variados.

Teniendo en cuenta lo anterior, el análisis se ha basado en la definición del mexicanismo facilitada por Company (2007: 28-29), ampliada al ámbito americano. Así, se entenderá al americanismo lingüístico como "el conjunto de voces, formas o construcciones que son caracterizadoras del habla urbana, popular o culta, o ambas, de América y cuyo uso muy frecuente y cotidiano distancia la variedad americana respecto del español peninsular"; en consecuencia, se interpretará a los elementos aislados asociados con las unidades mencionadas como americanismos correspondientes a los diferentes niveles de la lengua. La elección de esta propuesta se debe a lo apropiada que resulta para abordar las manifestaciones lingüísticas desde una perspectiva histórica, pues es posible considerar desde este enfoque como parte de una categoría a distintos elementos en la medida en que su empleo está determinado geográficamente en una sincronía específica.

De esta condición, se desprenden por lo menos tres principios metodológicos de suma relevancia para el estudio diacrónico de los americanismos léxicos o voces caracterizadoras del habla de América (Ramírez Luengo, en prensa):

1) La consideración del uso -y no de la etimología- como criterio único permite reconocer de manera más certera qué unidades léxicas son americanismos y establecer además la oposición con los indigenismos ${ }^{4}$, elementos con los cuales coinciden en algunos casos, pero que constituyen una categoría diferente .

2) El carácter dinámico-histórico de esta categoría obedece a su delimitación espacial y cronológica. La valoración como americanismos de ciertas voces no se mantiene estable, "sino que puede variar a lo largo de la historia, dependiendo de los procesos de expansión o reducción geográfica que experimenten" (Ramírez Luengo 2012: 398), esto es, de los procesos de extensión léxica ${ }^{6}$ que las afecten.

3) Las diferentes formas de cumplir con la definición propuesta por Company implican la existencia de distintos tipos de americanismos.

4 El indigenismo se sustenta en la procedencia de los términos. Es un 'vocablo, giro, rasgo fonético o semántico que pertenece a alguna lengua indígena de América o proviene de ella' (DLE 2014: s. v. indigenismo).

5 Este deslinde permite evitar las confusiones derivadas de aplicar criterios que conjuntan origen y uso, los cuales conducen a apreciaciones erróneas, como valorar voces generales como americanismos -por ejemplo, chocolate o tomate- o dejar de lado términos de uso exclusivo en América -tal es el caso de ahorita.

6 Aunque esta expresión también remite a la ampliación semántica de un término, en este estudio, será entendida en el sentido expresado por Ramírez Luengo (2014: 4), como "todo proceso (histórico) de modificación en la distribución geográfica de una voz, sea de expansión (generalización) o de reducción (dialectalización)”. 
Se infiere de este modo que no todos los vocablos "resultan propios de América de la misma manera” (Ramírez Luengo 2014: 3). Partiendo de esta condición, es posible establecer tres subtipos de americanismos léxicos (Company 2010: XVII): de frecuencia, voces compartidas en forma y significado con el español peninsular, pero que muestran en América una mayor frecuencia de uso; puros, voces empleadas en el español americano inexistentes en el español peninsular, y semánticos, voces compartidas formalmente con el español peninsular que han desarrollado en América valores semánticos propios.

Tras identificar las voces susceptibles de ser consideradas americanismos por corresponder a los tipos descritos antes, se verificó su estatus en los diccionarios de las academias (DLE 2014; DAMER 2010; DM 2010), en diccionarios históricos y etimológicos (DCECH 1980-1991; Company y Melis 2002), así como en obras lexicográficas y diccionarios sobre voces locales (DEM 2010; Morínigo 1998; Santamaría 1959, entre otros). Para constatar el empleo diferenciado de los términos, se consultó tanto el Corpus Diacrónico del Español (CORDE) como el Léxico hispanoamericano 1493-1993 (LEXHISP). En las búsquedas, se tuvieron en cuenta todas las posibilidades gráficas de los términos: con o sin mayúsculas, tilde o $h$ inicial -en palabras comenzadas en vocal-, así como las distintas alternativas entre sibilantes $-s, c, z$ y $c$ - o bien entre $b$ y $v$ o $g$ y $j$.

En el caso de las voces etiquetadas como americanismos de frecuencia, se contabilizaron los registros encontrados en CORDE, repositorio que constituyó el corpus de referencia para estos casos. Sin embargo, dada la desproporción entre el volumen de términos procedentes de obras peninsulares con relación a los incluidos en obras producidas en el Nuevo Mundo, fue necesario calcular además su frecuencia relativa con base en el total de palabras contenidas en los documentos españoles o americanos de los siglos XVII y XVIII (TABLA 3). El criterio para normalizar los datos fue "casos por millón de palabras" (CMP).

\begin{tabular}{|l|c|}
\hline Procedencia & Total de Palabras \\
\hline España & 38853728 \\
\hline Hispanoamérica & 10355681 \\
\hline
\end{tabular}

Tabla 3. Voces peninsulares e hispanoamericanas en CORDE (1601-1800).

\section{Americanismos léxicos en 18 textos veracruzanos del siglo XVII}

Con base en los criterios expuestos antes, se extrajeron del léxico presente en los textos producidos en Veracruz entre 1650 y 1700 que se incluyen en los DLNEGM los siguientes ejemplos de términos que pueden ser considerados americanismos en la sincronía estudiada. 
Algunas notas sobre el léxico veracruzano en la época colonial (1650-1700). La presencia de americanismos...

\begin{tabular}{|l|c|l|}
\hline TIPO AMERICANISMO & CASOS & \multicolumn{1}{|c|}{ VoCES } \\
\hline De frecuencia & $4(20 \%)$ & almácigo, batea, holán, paila \\
\hline Puros & $7(35 \%)$ & chilchote, coyol, huipil, médano, naguas, tecomate, tinajera \\
\hline Semánticos & $9(45 \%)$ & doctrina, ingenio, ladino, loba, morena, pardo, prieto, rancho, tortilla \\
\hline TOTAL & $20(100 \%)$ & \\
\hline
\end{tabular}

Tabla 4. Ejemplos de americanismos en los DLNEGM producidos en Veracruz entre 1650 y 1700.

Respecto a la distribución tentativa de estos ejemplos -los cuales representan un primer acercamiento al español en uso en Veracruz en la sincronía propuesta para este estudio-, resulta pertinente señalar que coincide con las observaciones de Quirós García y Ramírez Luengo (2015: 192) sobre la variedad yucateca de los siglos XVII y XVIII, pues en ella los americanismos semánticos y puros representan la mayoría de las voces propias del Nuevo Mundo (45\% y 35\% respectivamente) en tanto que los americanismos de frecuencia conforman un grupo marginal (20\%). No se debe perder de vista esta característica, ya que podría arrojar luces sobre la configuración léxica ${ }^{7}$ de la variedad en estudios futuros.

\subsection{Americanismos de frecuencia}

Con relación a los términos diatópicamente marcados por su frecuencia de uso -voces comunes en ambos lados del Atlántico, pero que en determinada sincronía resultan más habituales en América (Company 2007: 31)-, se extrajeron los siguientes ejemplos que cumplen esta condición: almácigo, batea, holán y paila.

Almácigo. m. Mx, Gu, Ho, Ni, CR, Ch, Ar, Ur. ${ }^{8}$ 'Terreno donde se siembran y se crían las plantas que luego han de trasplantarse' (DAMER 2010; DM 2010: s. v. almácigo).

Luego que llegó a dicho rancho, fue a ver un almásigo que tenía sembrado de semilla de tavaco (Córdoba 1696; documento 104)

$7 \quad$ "Preferencia que muestra una determinada variedad por una de las posibles estrategias que se emplean a la hora de dialectalizar -o americanizar - el léxico" (Ramírez Luengo, en prensa). Las estrategias fundamentales, como muestran diversos estudios (Buesa Oliver y Enguita Utrilla 1992; Frago 1999; Franco Figueroa 1991), son la modificación, adaptación del léxico existente que da como resultado nuevas formas o significados, y la incorporación, introducción de elementos léxicos tomados de otros sistemas lingüísticos, por lo general, los idiomas originarios de América. Todas las variedades americanas recurren a ambas estrategias, pero no lo hacen en la misma proporción. Las distintas correlaciones entre los elementos modificados -voces patrimoniales-y los incorporados -por lo general, indigenismos- dan por resultado diferentes configuraciones léxicas que identifican a los dialectos del español en el continente.

8 Se incluyen las marcas diatópicas como aparecen en las obras consultadas. En este caso, el DAMER (2010) utiliza dos letras para designar a cada país, ya sea abreviatura o sigla $(G u, R D)$, pero el orden representa un eje nortesur, oeste-este, con el fin de mostrar las correspondientes isoglosas léxicas. En el DLE (2014), en cambio, se emplean desde abreviaturas de dos, tres o cuatro caracteres (Ec., Méx. o Guat.) hasta topónimos completos cuando son cortos $(\mathrm{Cuba})$ y las marcas siguen un orden alfabético. En la mayoría de las obras consultadas, los criterios son similares a los del DLE. 
Aunque en el DLE (2014: s. v. almáciga) aparece la forma femenina con un sentido similar -'lugar donde se siembran y crían los vegetales que luego han de trasplantarse'- en el ámbito hispanoamericano predomina la forma masculina con una frecuencia relativa de 1.44 frente a 0.23 en España.

Batea. f. 'Artesa, generalmente redonda, que sirve para lavar y otros usos' (DLE 2014; DAMER 2010; DM 2010; DEM 2010: s. v. batea).

Dos bateas de labar, viejas (Veracruz 1655; documento 77)

Si bien presenta el mismo significado en los diccionarios consultados, la diferencia de frecuencia relativa de esta voz en los documentos americanos y en los peninsulares es contundente: 2.22 en el primer caso y 0.02 en el segundo.

Holán. f. 'Adorno compuesto por una tira de tela, que rodea ciertas prendas de vestir, especialmente femeninas y en algunos trajes regionales, plegado y cosido por la parte superior, y suelto o al aire por la inferior' (DM 2010; DEM 2010: s. v. holán).

Una saya de holán de Holanda plateada (Veracruz 1655; documento 77)

Al consultar el término en el DLE (2014: s. v. holán), se señala que se trata de un mexicanismo y remite al usuario a la voz faralá (\| volante). Este indicio de empleo diferenciado se confirma al comparar la frecuencia relativa de la palabra en textos producidos en el Nuevo Mundo y en España, la cual corresponde a 0.39 y a 0.20 , respectivamente.

Paila. f. 'Vasija grande de meta, redonda y poco profunda' (DLE 2014: s. v. paila).

Una paila mediana de cobre (Veracruz 1655; documento 77)

El DM (2010: s. v. paila) es más específico: en él se muestra que este término hace referencia a un 'recipiente en forma de círculo, de boca ancha, más hondo que la sartén y de fondo curvado, que se emplea para cocinar alimentos o tostar granos', o bien a un 'recipiente metálico de forma parabólica, con la boca muy ancha y pared alta, usado principalmente para cocer el jugo de la caña de azúcar'. En este caso, además dela especialización semántica del término en el Nuevo Mundo, se registra una muy notable diferencia en su frecuencia relativa: 0.57 en los documentos hispanoamericanos y 0.07 en los peninsulares.

Este ejemplo ilustra un problema derivado de la taxonomía de los americanismos léxicos, el hecho de que en muchas ocasiones los subtipos se traslapan. Decidir qué rasgo es el más prominente conlleva una alta carga de subjetividad que deberá ser acotada en estudios posteriores para definir de manera cada vez más precisa el concepto de americanismo y la clasificación de los elementos incluidos en esta categoría de análisis. 


\subsection{Americanismos puros}

En el caso de esta clase de términos definidos por Company (2007: 30) como "voces de uso general en América inexistentes -o al menos poco conocidas- en España", se detectaron siete ejemplos: chilchote, coyol, huipil, médano, naguas, tecomate y tinajera.

Chilchote (Del náhuatl chilli). m. Méx. 'Chile muy picante' (DLE 2014; DM 2010; DEM 2010: s. v. chilchote).

Sierta yerba que criaba y daba uñas a modo de chilchotes (Orizaba 1675; documento 89)

Coyol (Del náhuatl coyolli). 1. m. C. Rica, El Salv., Guat., Hond., Méx. y Nic. 'Palmera de mediana altura, de cuyo tronco, provisto de espinas largas y fuertes, se extrae una bebida que fermenta rápidamente'. 2. m. 'Fruto del coyol' (DLE 2014: s. v. coyol).

Un rosario de coyol con bordillas de seda (Veracruz 1655; documento 77)

Huipil (Del náhuatl huipilli). m. El Salv., Guat., Hond. y Méx. 'Especie de blusa adornada propia de los trajes indígenas'. (DLE 2014; DM 2010; DEM 2010: s. v. huipil).

Traýa vestido un huipil ordinario destos de que usan las indias (Xalapa 1674; documento 87)

Médano (Del ár. hisp. máydan, y éste del ár. clás. maydān 'explanada’ [de arena]; cf. port. médão). 1. m. 'Duna'. 2. m. 'Montón de arena casi a flor de agua, en un paraje en que el mar tiene poco fondo' (DLE 2014; DEM 2010: s. v. médano).

Desde el collegio de la Compañía de Jesús azta los médanos (Orizaba 1675; documento 89)

Naguas (Voz taína).f. 'Saya interior de tela blanca' (DLE 2014: s. v. naguas). 1. f. pl. $M x, R D, G u, H o$, $E S, B o, C o, E c$ 'Prenda interior femenina, similar a una falda que se lleva debajo de ésta'. 2. f. $M x$, $E S, N i, C R$. 'Saya o falda' (DAMER 2010: s. v. naguas).

Unas nagoas de crea picadas con randilla de pita (Veracruz 1655; documento 77)

Tecomate (Del náhuatl tecomatl). 1. m. El Salv., Guat., Hond., Méx. y Nic. 'Especie de calabaza de corteza dura, de la cual se hacen vasijas'. 2. m. El Salv., Guat., Hond., Méx. y Nic. 'Vasija hecha con tecomate'. (DLE 2014: DM, 2010: s. v. tecomate).

Un tecomate de Peribán9 (Veracruz 1655; documento 77)

9 Municipio de Michoacán. 
Tinajera. f. 'Sitio o lugar donde se ponen o empotran las tinajas' (DLE 2014: s. v. tinajera). f. Pa. 'Mueble donde se ponen las tinajas' (DAMER 2010: s. v. tinajera).

Una tinagera de zedro, nueba (Veracruz 1655; documento 77)

Cabe señalar que mientras los ejemplos de americanismos de frecuencia presentados antes son endohispánicos ${ }^{10}$, la procedencia de estas voces es etimológicamente heterogénea. Se detectaron, de entrada, dos términos provienen del léxico patrimonial, médano y tinajera. Respecto al primero, Frago (1999: 137) aclara que médano 'duna' es una voz común en las hablas canarias, las cuales habrían sido un "importante foco de difusión de su expansión americana” y hace hincapié en su temprana documentación en un texto de 1553 sobre las dunas del litoral veracruzano; por su parte, Corominas y Pascual (DCECH 1980-1991: s. v. médano) sugieren que se trata de un lusismo, pues el registro más antiguo del vocablo está en portugués, aunque no descartan la posibilidad de documentaciones previas.

En cuanto a tinajera, 'mueble donde se ponen las tinajas' (DAMER 2010: s. v. tinajera), sólo aparece en los diccionarios de las academias; sin embargo, en Santamaría (1959: s. v. tinajero), se asocia a la forma masculina tinajero con la acepción 'sitio donde se tienen las tinajas, jarros y demás enseres relativos al servicio del agua potable en casa’ y se aclara que también se utiliza en Canarias. La vinculación de ambos vocablos con el habla del archipiélago canario concuerda con la tendencia descrita por Ramírez Luengo (2007: 73) de usar “dialectalismos léxicos -por lo general, canarismos y andalucismos- para denominar las nuevas realidades americanas". Con el fin de constatar el estatus de estas voces, además de las obras lexicográficas citadas, se consultaron las bases de datos CORDE y LEXHISP ${ }^{11}$.

Por otro lado, hay cinco voces indígenas: cuatro de origen náhuatl -chilchote 'chile picoso', coyol 'palmera', huipil 'vestimenta indígena’y tecomate 'vasija' - y una de origen taíno -naguas 'falda'-. No sorprende el origen preponderantemente náhuatl de estos términos, dada la función de lengua general que tuvo este idioma durante la época colonial ${ }^{12}$, como tampoco la presencia del vocablo taíno, pues los primeros contactos entre los colonizadores y los nativos de América se producen en las Antillas, lo cual "determina, como es natural, que sean las lenguas de estas islas -en especial, el taíno y el arahuaco- las que facilitan los términos más antiguos” (Ramírez Luengo 2007: 76).

10 Montes Giraldo (1995: 153) denomina americanismos endohispánicos a las voces propias de América que proceden del fondo hispánico.

11 Médano se encuentra en CORDE en 14 textos producidos entre los siglos XVII y XVIII en el Nuevo Continente y en cinco procedentes de España, pero cuatro de ellos son de temática americana: Segunda parte de la Crónica general de Indias (Francisco López de Gómara 1553), Crónica de la Nueva España (Francisco Hernández de Salazar, 1560), Historia verdadera de la conquista de la Nueva España (Bernal Díaz del Castillo 1568-1575) y Descripción de las costas de California (Fray Íñigo Abbad y Lasierra 1783). Con respecto a tinajera, únicamente hay registro de un texto de 1638 en LEXHISP, resguardado en el Archivo Histórico de Hidalgo del Parral, Chihuahua.

12 La sociedad de aquel momento no está en contacto con la inmensa variedad lingüística del continente, sino tan sólo con algunos idiomas, como el náhuatl o el quechua, los cuales adquieren carácter de lenguas generales y median la comunicación entre colonos e indígenas. De ahí que no todas las lenguas amerindias contribuyan en la misma proporción al "enriquecimiento léxico del español” (Ramírez Luengo 2007: 76-77). 
Queda destacar, por último, el grado de integración de estas voces en la variedad y la sincronía estudiadas. Como observa Morínigo (1959: 17), el hecho de que no aparezcan acompañadas de comentarios que las señalen como términos ajenos al español ni de ningún tipo de acotaciones es un indicio de que ya forman parte del habla cotidiana y apunta hacia un alto grado de asimilación en el dialecto de los productores de los textos.

\subsection{Americanismos semánticos}

Estas voces se caracterizan por desarrollar valores semánticos en América distintos de los peninsulares (Company 2007: 33). De acuerdo con Ramírez Luengo (2011: 10), se trata de palabras procedentes del léxico patrimonial, relacionadas sobre todo con el entorno social. En gran parte de los americanismos semánticos encontrados en los DLNEGM, se constató esta condición. Los ejemplos que corresponden a dicho grupo son los siguientes: doctrina, ingenio, ladino, loba, morena, pardo, prieto, rancho y tortilla.

Doctrina. f. 'En América, distrito eclesiástico servido por un sacerdote expresamente nombrado para adoctrinar a la población indígena' (DLE 2014: s. v. doctrina). f. 'Curato colativo servido por regulares' (Santamaría 1959: s. v. doctrina).

En el pueblo de Santa Ana de la doctrina de San Joan Coscomatepeque, en beyte y un dias del mes de otubre (Santa Ana 1675; documento 88)

Ingenio. m. ingenio de azúcar. 1. m. ‘Conjunto de aparatos para moler la caña y obtener el azúcar’. 2. m. 'Finca que contiene el cañamelar y las oficinas de beneficio' (DLE 2014: s. v. ingenio). 'Hacienda de caña donde se fabrica azúcar' (Morínigo 1998: s. v. ingenio).

Aviendo estado en el ingenio de la Santíssima Trinidad que es del capitán don Francisco de la Higuera (Xalapa 1674; documento 87)

Ladino, na. adj. 'Mestizo o indio que habla español o tiene costumbres ajenas a su comunidad de origen' (DM 2010: s. v. ladino). adj. y s. 'Por contraposición al indio que conserva su lengua y sus costumbres, blanco, mestizo o indio que habla español o tiene costumbres occidentales' (DEM 2010: s. v. ladino).

Y que, como a las dies de la noche, poco más o menos, trajeron presso a dicha prission a un yndio ladino llamado Juan (Xalapa 1681; documento 94)

Lobo, ba. adj. 'En la América colonial, nacido de india y negra o de negro e india' (DLE 2014: s. v. lobo).

Juana de la Candelaria mulata loba, soltera, libre, que se ha criado en cassa de su tía Mariana (Orizaba 1655; documento 86) 
Moreno, na (De moro y -eno). adj. coloq. 'Dicho de una persona: De raza negra'. adj. Cuba. 'mulato' (nacido de negro y blanca, o de blanco y negra) (DLE 2014: s. v. moreno). 'Por eufemismo, se llama así al negro' (Santamaría 1959: s. v. moreno).

En quanto a la prisión de Adriana, morena libre (Veracruz 1655; documento 77)

Pardo, da (Del lat. pardus 'leopardo', por alusión a su color, y éste del gr. đá $\rho \delta o \varsigma$ párdos). adj. Ant., Arg., Ec., Hond., Méx., Perú, Ur. y Ven. p. us. 'Mulato' (nacido de negro y blanca, o de blanco y negra) (DLE 2014; DAMER 2010; Santamaría 1959: s. v. pardo).

Andrés de Vega, pardo libre, vezino de esta ciudad (Veracruz 1691; documento 101)

Prieto, ta (De apretar). adj. Cuba. 'Dicho de una persona: De raza negra'. Méx. 'Dicho de una persona: De piel morena' (DLE 2014; DAMER 2010; DEM 2010; Santamaría 1959: s. v. prieto). adj. despect. 'Referido a alguien de piel morena' (DM 2010: s. v. prieto).

Que echasse aquellos polvos en la comida o bebida que havía que comer o beber María, mulata prieta (Orizaba 1655; documento 86)

Rancho (De rancharse). m. supran. 'Hacienda ganadera o agrícola' (DEM 2010: s. v. rancho). m. 'Terreno relativamente extenso dedicado al cultivo y a la cría de animales, general provisto de uno o más edificios donde viven sus dueños y trabajadores, y donde se guarda le herramienta, el grano, etcétera' (DEM 2010: s. v. rancho).

Aviendo llegado este declarante a un rrancho que dicho su padre tiene en dicha jurisdizión, y como a las ocho de la noche, poco más o menos, luego que llegó a dicho rancho, fue a ver un almásigo que tenía sembrado de semilla de tavaco (Córdoba 1696; documento 104)

Tortilla (Del dim. de torta). f. supran. 'Alimento en forma circular y aplanada, para acompañar la comida, que se hace con masa de maíz, hervido en agua con cal; se cuece generalmente en comal' (DM 2010: s. v. tortilla).

Entonces en un apossento, le sacó dos tortillas de maýs (Xalapa 1674: documento 87)

Los vocablos anteriores son adaptaciones de términos patrimoniales a la realidad americana mediante procesos de deslizamiento semántico, entre los cuales, la metonimia y la metáfora son los más comunes. El primer mecanismo implica una "conexión entre dos conceptos, de alguna manera contiguos" (Espinosa Elorza 2009: 159). Doctrina es un claro ejemplo. Según el Diccionario de términos coloniales (Velázquez 1944: s. v. doctrina), dicha palabra designaba al 'distrito eclesiástico a cargo de un convento o de un religioso, compuesto generalmente por un pueblo cabecera y varios pueblos de visita. Como puede apreciarse, el territorio establecido para difundir la doctrina (cristiana) adquiere el nombre de ésta. Por medio de dicho mecanismo, se denomina una realidad distinta de la española, ligada en este caso a una estructura fundamental para la evangelización de la población indígena. 
El segundo proceso de deslizamiento semántico, la metáfora, consiste en "una proyección entre dos estructuras conceptuales, basada en la semejanza” (Espinosa Elorza 2009: 188). La voz lobo 'nacido de india y negra o de negro e india' (DLE 2014: s. v. lobo) permite ilustrar este mecanismo. Este término podría ser "la evolución semántica del cánido -por el color de su pelaje- al hombre de piel oscura" (Alvar 1998: 324). El ejemplo se vincula en particular con lo que Lakkoff y Johnson llaman metáfora zoomórfica (Espinosa Elorza 2009: 170), la cual consiste en la proyección del dominio animal en el humano.

Según Corominas y Pascual (DCECH 1980-1991: s. v. pardo), el vocablo pardo 'mulato' comparte este rasgo, pues se interpreta "como un adjetivo expresivo de la principal diferencia que separa al leopardo del león, a saber, sus manchas de color negruzco”. En la misma línea, tanto prieto 'dicho de una persona: de piel morena' (DLE 2010: s. v. prieto) como moreno 'dicho de una persona: de raza negra', 'mulato' (DLE 2010: s. v. moreno) también pueden ser calificativos de animales $^{13}$ (Alvar 1998: 316).

Designar los cruces humanos con los nombres de los de animales no resulta extraño; en las denominaciones relacionadas con el mestizaje es común el empleo de estos recursos. La diversidad de tipos humanos generada por el fenómeno exigió un esfuerzo de categorización que se valió de tales medios. La nomenclatura resultante ${ }^{14}$ constituye un campo muy rico para el estudio del léxico colonial que hasta el momento ha sido explorado por pocos autores (Aguirre Beltrán 1989 [1946]; Moreno Navarro 1969; Alvar 1987 y 1998).

Un último término ligado a esta temática es ladino blanco, mestizo o indio que habla español o tiene costumbres occidentales' (DEM 2010: s. v. ladino), el cual, a diferencia de las voces anteriores, no se vincula con una condición étnica en sí, sino con una capacidad asociada a dicha condición. De hecho, el sentido observado en los DLNEGM no coincide con la noción de 'mestizo' que predomina en las acepciones recogidas en el DLE y el DAMER (2014; 2010: s. v. ladino) ni con el valor que se presenta como básico en la primera obra lexicográfica: 'astuto, sagaz, taimado' (DLE 2010: s. v. ladino).

Tal sentido parece guardar, en cambio, una relación más estrecha con la aplicación original del término, según Corominas y Pascual (DCECH 1980-1991: s. v. ladino), la referencia en la Edad Media a la lengua romance por oposición a la arábiga y al moro que hablaba aquel idioma; la primera documentación del vocablo lo constata: 'moro tan ladino que semejava christiano' (finales del siglo XIII, Primera crónica general). Así, el valor relativo a la facultad de hablar una lengua diferente a la materna o de adaptarse a costumbres ajenas concuerda con el empleo más antiguo. Esta coincidencia quizá obedece al uso de una analogía anclada en situaciones de contacto intercultural y en las aptitudes que conllevan.

Además de las voces ligadas a la organización social colonial, se detectaron dos elementos relacionados con la dinámica económica del virreinato: rancho e ingenio. Derivado del verbo de origen francés ranchar o ranchearse 'alojarse', el primer término se aplica en México a 'una

13 Ambos términos tienen, por otro lado, implicaciones pragmáticas; tal y como indican las acotaciones lexicográficas, prieto sería una referencia despectiva a 'alguien de piel morena' (DM 2010: s. v. prieto) y moreno, un eufemismo de 'negro' (Santamaría 1959: s. v. moreno).

14 Desde fines del siglo XVI y a lo largo del XVII, se desarrolló en la Nueva España una terminología basada en el color de la piel que en el siglo XVIII llega a su punto máximo con las colecciones de Cuadros del mestizaje americano, expresión estética también conocida como "pintura de castas" (Moreno Navarro 1969: 209-209). 
hacienda de mediano tamaño' (DCECH: s. v. rancho), mientras que en la mayor parte del continente se conserva el sentido primitivo de habitación pequeña y pobremente construida. En los siglos XVI y XVII, la palabra era bien conocida en los países de habla hispana y designaba desde entonces "cualquier tipo de vivienda provisional” (Ramírez Luengo 2011: 12). Sin embargo, la especialización mexicana, al parecer, ya se había producido para la segunda mitad del siglo XVII, como se aprecia en los DLNEGM.

El segundo elemento designa 'por antonomasia, el ingenio de azúcar', según Santamaría (1959: s. v. ingenio). De acuerdo con los datos de CORDE, probablemente ya predominaba este sentido de la palabra en América en el siglo XVIII, puesto que no hay registros hispanoamericanos de la frase ingenio de azúcar en esta centuria y tan sólo existen cuatro en la anterior, pese a ser una institución relevante en la economía colonial, introducida al continente para asegurar el sustento de los encomenderos (Von Wobeser 1988: 6-8).

Se encontró en este grupo una voz relacionada con la alimentación: tortilla 'alimento en forma de torta circular y aplanada, elaborado con masa de maíz o trigo' (DAMER 2010: s. v. tortilla). Si bien el término mantiene el rasgo más prominente del valor original, la forma circular, ha sufrido una serie de alteraciones -que van desde los ingredientes y la consistencia hasta la forma de preparación- para designar un elemento propio de la realidad americana.

\section{Distribución de los americanismos léxicos por tipos y campos semánticos}

Además de las descripciones particulares, es importante determinar desde la primera aproximación a una variedad cómo se correlacionan los elementos que la caracterizan, en este caso, los correspondientes al nivel léxico. En la siguiente tabla, se muestra la distribución de las voces analizadas por el tipo de americanismo al que pertenecen. (TABLA 5)

\begin{tabular}{|c|c|c|c|}
\hline TIPO DE AMERICANISMO & CAsos & $\%$ & VOCES \\
\hline Semánticos & 9 & 45 & $\begin{array}{l}\text { doctrina, ingenio, ladino, loba, morena, pardo, prieto, } \\
\text { rancho, tortilla }\end{array}$ \\
\hline Puros & 7 & 35 & $\begin{array}{l}\text { chilchote, coyol, huipil, médano, naguas, tecomate, } \\
\text { tinajera }\end{array}$ \\
\hline De frecuencia & 4 & 20 & almácigo, batea, holán, paila \\
\hline TOTAL & 20 & 100 & \\
\hline
\end{tabular}

Tabla 5. Distribución de americanismos léxicos por tipo.

Conjuntando las propuestas de Mejías (1980: 22) y Polo Cano (2005: 189), se han establecido también los campos semánticos o ámbitos en que se agrupan las unidades abordadas en este estudio. Es notable, de entrada, la ausencia de voces alusivas a la flora y la fauna, además de la preponderancia general de los términos vinculados con aspectos sociales y culturales: 
a) Americanismos de frecuencia

- Enseres/utensilios: batea, paila

- Prendas de vestir: holán

- Agricultura: almácigo

b) Americanismos puros

- Enseres/utensilios: tecomate, tinajera

- Prendas de vestir: huipil, naguas

- Alimentación: chilchote, coyol

- Clima, geografía: médano

c) Americanismos semánticos

- Organización social: doctrina, ladino, lobo, moreno, pardo, prieto

- Industria/construcción: ingenio

- Agricultura: rancho

- Alimentación: tortilla

En el caso de los americanismos semánticos, el grupo más numeroso, se verifica la descripción de Ramírez Luengo (2011: 10), quien señala que, por lo general, son voces patrimoniales, relacionadas sobre todo con el contexto social. En contraste, sólo se encontró un elemento -un americanismo puro- vinculado con el entorno físico: médano 'duna'. La distribución general de los términos por campos semánticos se observa enseguida. (TABLA 6)

\begin{tabular}{|l|c|c|l|}
\hline CAMPO SEMÁNTICO & CASOS & $\%$ & \multicolumn{1}{|c|}{ VOCES } \\
\hline Organización social & 6 & 30 & doctrina, ladino, lobo, moreno, pardo, prieto \\
\hline Enseres/utensilios & 4 & 20 & batea, paila, tecomate, tinajera \\
\hline Prendas de vestir & 3 & 15 & huipil, holán, naguas \\
\hline Alimentación & 3 & 15 & chilchote, coyol, tortilla \\
\hline Agricultura & 2 & 10 & almácigo, rancho \\
\hline Clima/geografía & 1 & 5 & médano \\
\hline Industria/construcción & 1 & 5 & ingenio \\
\hline TotAL & 20 & 100 & \\
\hline
\end{tabular}

Tabla 6. Distribución de americanismos léxicos por campos semánticos 


\section{Conclusiones}

Con base en los datos obtenidos en este primer acercamiento al español en uso en Veracruz durante la segunda mitad del siglo XVII, se pueden establecer algunas conclusiones:

1) La presencia de los tres tipos de americanismos léxicos descritos por Company (2010: XVII; 2007: 28-29) en los textos incluidos en los DLNEGM seleccionados para el análisis apunta hacia un proceso de dialectalización en marcha en este nivel de la lengua.

2) La preponderancia de los americanismos endohispánicos sobre los elementos procedentes de otros sistemas lingüísticos, en una proporción de 15 términos patrimoniales frente a cinco indigenismos -chilchote, coyol, huipil, nahuas, tecomate-, es indicio de una posible preferencia por la estrategia de adaptación sobre la estrategia de incorporación y, por tanto, de una configuración léxica particular de la variedad estudiada.

3) No sorprende el predominio de las voces de origen náhuatl entre los indigenismos detectados, dada su función como lengua general de la Nueva España, ni la presencia del término taíno naguas por el contacto con las Antillas. Cabe destacar que el hecho de que dichos elementos no aparezcan acompañados de acotaciones muestra un grado de asimilación significativo de estos vocablos en el dialecto de los productores de los textos.

4) Sobresale asimismo la presencia mayoritaria de los americanismos semánticos, los cuales representan $45 \%$ de las voces analizadas. El campo semántico más relevante es "organización social" al que pertenece $30 \%$ de los términos; en él se incluyen algunas denominaciones relativas a la condición étnica: ladino, lobo, moreno, pardo, prieto. No hay que perder de vista que Veracruz es una zona de grandes asentamientos de afromestizos.

5) No se detectaron primeras dataciones entre los ejemplos tomados para el análisis.

Aunque este trabajo no pretendía ser exhaustivo, se lograron vislumbrar algunas tendencias del léxico de la variedad analizada, entre ellas, la exigua presencia de indigenismos y de términos relacionados con el entorno natural, y la preponderancia de las denominaciones vinculadas con el contexto social, en particular las alusivas a la condición étnica, las cuales conforman un campo con gran potencial para el estudio del léxico colonial.

\section{Referencias bibliográficas:}

Alvar, M. (1987). Léxico del mestizaje en Hispanoamérica. Madrid: Cultura Hispánica.

- (1998). Las castas coloniales en un cuadro de la Real Academia Española. Boletín de la Real Academia Española, LXXVIII, 307-338.

Aguirre Beltrán, G. (1989 [1946]). Obra Antropológica II. La población negra de México. Estudio etnohistórico. México: Universidad Veracruzana/ Instituto Nacional Indigenista/ Gobierno del Estado de Veracruz/ Fondo de Cultura Económica.

Buesa Oliver, T.; \& Enguita Utrilla, J. M. (1992). Léxico del español de América. Su elemento patrimonial e indígena. Madrid: MAPFRE. 
Company, C. (1994). Documentos lingüísticos de la Nueva España. Altiplano-Central. México: UNAM.

- (2007). El siglo XVIII y la identidad lingüística mexicana. Discurso de ingreso a la Academia Mexicana de la Lengua (10 de noviembre de 2005). México: UNAM/Academia Mexicana de la Lengua.

- (2010). Introducción. In Academia Mexicana de la Lengua: Diccionario de mexicanismos. México: Siglo XXI.

Company, C.; \& Melis, C. (2002). Léxico histórico del español de México. Régimen, clases funcionales, usos sintácticos, frecuencias y variación gráfica. México: UNAM.

[CORDE]: Real Academia Española. Corpus Diacrónico del Español. Disponible en: <http://corpus.rae.es/ cordenet.html> [Consulta: abril, 2017]

[DAMER]: Asociación de Academias de la Lengua (2010). Diccionario de Americanismos. Disponible en: <http://www.asale.org/recursos/diccionarios/damer=> [Consulta: abril, 2017]

[DCECH]: Corominas, J.; Pascual, J. A. (1980-1991). Diccionario crítico etimológico castellano e hispánico. Madrid: Gredos.

[DEM]: El Colegio de México (2010). Diccionario del Español de México. Disponible en: <http://dem. colmex.mx> [Consulta: abril, 2017]

[DM]: Academia Mexicana de la Lengua (2010). Diccionario de mexicanismos. México: Siglo XXI EditoresAcademia Mexicana de la Lengua.

[DLE]: Real Academia Española (2014). Diccionario de la lengua española (23a ed.). Disponible en: <http:// www.rae.es> [Consulta: abril, 2017]

Frago, J. A. (1999). Historia del español de América. Madrid: Gredos.

Franco Figueroa, M. (1991). Léxico hispanoamericano de los siglos XVI y XVII en fuentes de América Central $y$ de la Nueva España. Sevilla: Universidad de Sevilla.

Espinosa Elorza, R. (2009). El cambio semántico. In E. de Miguel (Ed.), Panorama de la lexicología (pp. 159-188). Barcelona: Ariel.

Instituto Nacional para el Federalismo y el Desarrollo Municipal (INAFED) (2010). Enciclopedia de los municipios y delegaciones de México. Disponible en: <http://www.inafed.gob.mx/work/enciclopedia/> [Consulta: mayo, 2017]

[LEXHISP]: Boyd-Bowman, P. (2003). Léxico hispanoamericano1493-1993. Nueva York: Hispanic Seminary of Medieval Studies. Disponible en: <http://textred.spanport.lss.wisc.edu> [Consulta: mayo, 2017]

Lope Blanch, J. M. (1970). Las zonas dialectales de México. Nueva Revista de Filología Hispánica, 19, 1-11.

López-Beltrán, C. (2008). Sangre y temperamento: pureza y mestizajes en las sociedades de castas americanas. In F. Gorbach, \& C. López-Beltrán (Eds.), Saberes locales: ensayos sobre historia de la ciencia en América Latina. Zamora, Michoacán: El Colegio de Michoacán.

Mejías, H. (1980). Préstamos de lenguas indígenas en el español americano del siglo XVII. México: UNAM.

Melis, C.; \& Rivero Franyutti, A. (2008). Documentos lingüísticos de la Nueva España. Golfo de México. México: UNAM.

Montes Giraldo, J. J. (1995). Dialectología general e hispanoamericana. Bogotá: Instituto Caro y Cuervo.

Moreno de Alba, J. G. (1976). Zonas dialectales de Tabasco y Veracruz. Estudio léxico. Nueva Revista de Filología Hispánica, 25 (2), 332-352.

Moreno Navarro, I. (1969). Un aspecto del mestizaje americano: el problema de la terminología. Revista Española de Antropología Americana, 4, 201-218.

Morínigo, M. A. (1959). Indigenismos americanos en el léxico de Lope de Vega. In Programa de Filología Hispánica. Buenos Aires: Nova. 
. (1998). Nuevo Diccionario de Americanismos e Indigenismos. Buenos Aires: Claridad.

Polo Cano, N. (2005). Algunos indigenismos léxicos en el español de Guatemala del siglo XVIII. In A. García Lenza, \& A. Rodríguez Barreiro (Coords.), Res Diachronicae Virtual, 4, 185-102. Disponible en: $<$ https://resdi.net/volumen-iv/> [Consulta: abril, 2017]

Quirós García, M.; \& Ramírez Luengo, J. L. (2015). Observaciones sobre el léxico del español de Yucatán (1650-1800). Revista de Filología Española, 95, 183-210.

Ramírez Luengo, J. L. (2007). Breve historia del español de América. Madrid: Arco/Libros.

- (2011). Apuntes sobre el español altoperuano en el siglo XVIII. Cuadernos de Ilustración y Romanticismo, 17, 1-22.

. (2012). El léxico en los procesos de dialectalización del español americano: el caso de la Bolivia andina. Cuadernos del Instituto de Lengua Española, 7, 393-404.

- (2014). Cómo el español de España genera americanismos: a propósito del americanismo puto 'homosexual.' Lengua y Habla, 18, 1-12.

. (en prensa). Aspectos metodológicos para el estudio histórico del léxico americano: conceptos, ejemplificación y tareas para el futuro. Moenia, 23.

Santamaría, F. J. (1959). Diccionario de mejicanismos. México: Porrúa.

Velázquez, C. (1944). Diccionario de términos coloniales. San José de Costa Rica: Universidad de Costa Rica.

Von Wobeser, G. (1988). La industria azucarera en la época colonial. México: SEP-UNAM. 
